\title{
Perfil dermatoglífico de jugadores de handball de la UACH: una primera aproximación.
}

Dermatoglyphic profile of UACH handball players: a first approach.

\section{Fernández Aljoe, Rahndol ${ }^{1}$, Islas Guerra, Samuel Alfredo', Gastélum-Cuadras, Gabriel ${ }^{1}$.}

${ }^{1}$ Universidad Autónoma de Chihuahua, Facultad de Ciencias de la Cultura Física. Circuito Universitario Campus II, Fracc. Campo Bello, 31124, Chihuahua México. Tel. +52 6141589900 ext. 4639

\section{CORRESPONDENCIA}

\section{Gabriel Gastélum-Cuadras:}

gastelum@uach.mx

Universidad Autónoma de Chihuahua, Facultad de Ciencias de la Cultura Física.

\section{CÓMO CITAR}

Fernández, R., Islas, S., Gastélum, G. (2020) Perfil dermatoglífico de jugadores de handball de la UACH: una primera aproximación. Revista de Ciencias del Ejercicio, 15 (2), pp. 23-31. Recuperado de: revistafod.uanl.mx

\section{RESUMEN}

El propósito de este estudio fue conocer el perfil dermatoglífico y su relación con las potencialidades físicas de un grupo de jugadores de handball de la UACH de la ciudad de Chihuahua. La muestra fue escogida intencionalmente y compuesta por $(n=10)$ atletas masculinos. Se les realizó la toma de las huellas dactilares mediante el protocolo dermatoglífico propuesto por Cummins y Midlo (1961). Teniendo como resultado una mayor presencia del dibujo presilla (L), seguido del patrón verticilo (W) presentando el grupo de atletas estudiado predisposición a la velocidad y coordinación motora. En relación a los valores cuantitativos el Delta 10 (D10) y la Suma Total de Líneas Dermatoglíficas (SQTL), que se observan en este grupo muestran predisposición a la velocidad y la fuerza explosiva. Por otro lado, la fórmula dermatoglífica más encontrada fue la $\mathrm{L}>\mathrm{W}$ (40\%) y D10 (30\%) que se relacionan con velocidad, seguida de coordinación motora. Concluyendo que las potencialidades que más resaltan en este grupo de atletas son la velocidad y la coordinación motora potencialidades inherentes para el handball y los deportes colectivos, entre otros.

\section{ABSTRACT}

The purpose of this study was to know the dermatoglyphic profile and its relationship with the physical potential of a group of UACH handball players in the city of Chihuahua. The sample was intentionally chosen and composed of $(n=10)$ male athletes. The fingerprints were taken using the dermatoglyphic protocol proposed by Cummins and Midlo (1961). Having as a result a greater presence of the clip pattern $(\mathrm{L})$, followed by the verticyl pattern (W) presenting the group of athletes studied predisposition to speed and motor coordination. In relation to the quantitative values, Delta 10 (D10) and the Total Sum of Dermatoglyphic Lines (SQTL), which are observed in this group, show a predisposition to velocity and explosive force. On the other hand, the most commonly found dermatoglyphic formula was L> W (40\%) and D10 (30\%) that are related to speed, followed by motor coordination. Concluding that the potentialities that stand out in this group of athletes are the speed and motor coordination inherent potentials for handball and collective sports, among others.
Palabras clave: handball, atletas universitarios, potencialidades físicas, dermatoglifia, tecnología.
Key words: handball, university athletes, physical potentials, dermatoglyphs, technology. 


\section{Introducción}

La dermatoglifia es un método propuesto por Cummins y Midlo (1961), estos lo definieron a partir de dos elementos provenientes del griego, derma, que significa piel y glypho, relacionado con escritura y grabado (Hernández, 2017). Esta herramienta mide la propensión de las capacidades físicas innatas, a través del estudio de las huellas que se adquieren en el desarrollo fetal, basándose en la lectura de las marcas en las falanges de las manos y el desciframiento de sus diversas combinaciones Nodari-Júnior (2009) y Nodari y Heberle (2014). Por lo que, la combinación de genotipo y fenotipo hace mayor la posibilidad de orientarlos hacia una modalidad deportiva dada.Física y Readaptación Deportiva y el consolidado Doctorado en Ciencias de la Cultura Física que se ofrecen en la División de Posgrado, (así como de las diversas maestrías) donde surgen excelentes investigadores en el campo de la educación física, todo esto para conocer tanto la satisfacción, se ha dado un seguimiento semestre a semestre para conocer las actividades a las que se dedican y relacionadas con su formación académica.
A continuación, se comenta sobre los diferentes tipos de huellas. En primer término, tenemos al $\operatorname{Arco}(A)$ diseño caracterizado por la ausencia de deltas y que se compone solo de crestas que atraviesan la almohadilla digital transversalmente. La Presilla (L), por su parte es un diseño con un delta y núcleo, parcialmente cerrado en el que las crestas de la piel comienzan en un extremo del dedo y se acortan distalmente en relación con el otro sin acercarse al lugar donde se originan, existen dos tipos de presilla (LU) que tiende hacia el cubito y (LR) hacia el radio esta representa en alto rendimiento y se encuentra fundamentalmente en el dedo número dos de cada mano. El Verticilo (W y S), diseño de dos deltas, que se subdivide en $W$, el cual contiene un solo núcleo y el $\mathrm{S}$, que posee, igual al anterior, dos deltas con dos núcleos ligadas o unidas que forman una $\mathrm{S}$ o un doble verticilo o remolino (Figura 1).

En relación con la suma de la cantidad total de líneas (SQTL), esta se refiere a la sumatoria de las líneas de todos los dedos de las manos y la cantidad de líneas (QL) es el número de líneas de las crestas de la piel dentro del dibujo de cada dedo.

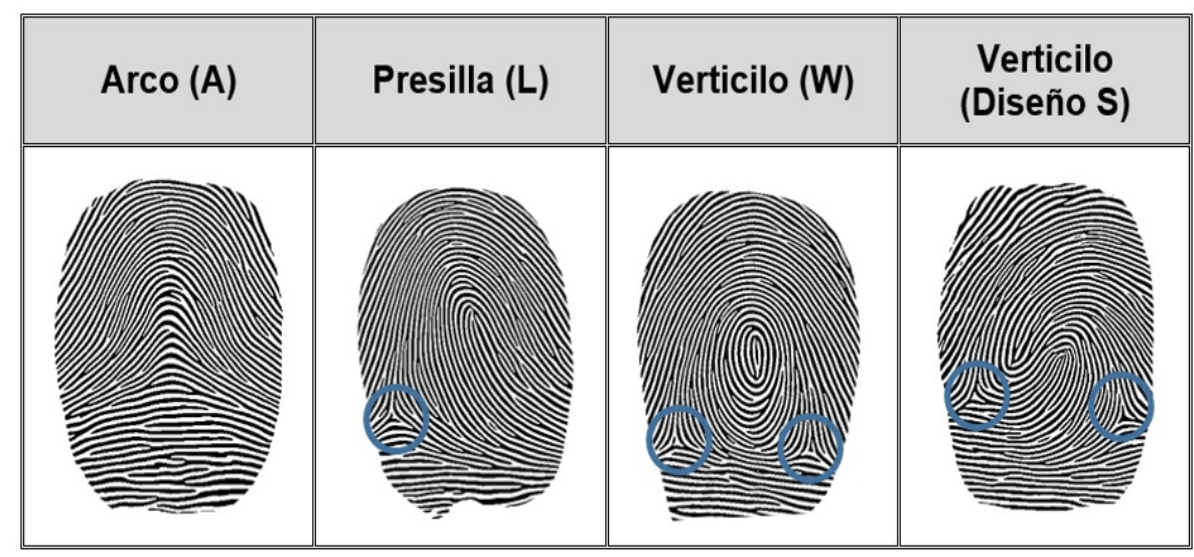

Figura 1. Patrones dermatoglíficos más comunes 
El delta (D10) es la suma de deltas de los dedos de ambas manos (Hernández y Naranjo, 2016). Por otra parte, los tipos de fórmulas digitales indican la representación de las diferentes combinaciones de dibujos en cada individuo: AL- presencia de arco y presilla; ALW- apariencia de Arco, presilla y verticilo; 10L- Diez presillas; LW- presilla y verticilo en el que el número de presilla es mayor o igual que 5; WL- El verticilo y la presilla donde el número de verticilos es mayor que 5 (Rodrigues y Fernandes, 2004).

En el deporte ya hace varios años se está utilizando la dermatoglífica como marcador genético, en la predicción de las dotaciones motoras de atletas en diferentes tipos de disciplinas y estos estudios se están usando para seleccionar deportistas con destacados rendimientos, con el propósito de estudiar y entender los mecanismos de distribución de los índices cuantitativos y cualitativos de la dermatoglífia (da Cunha, Pretto y Fernandes, 2006; Sena et al., 2012 y Nogueira et al., 2005). Estos autores han fundamentado la idea de que la impresión de la huella digital de las manos sirve para evaluar el potencial físico de los deportistas de handball.

La complejidad de los dibujos de las huellas dactilares puede comprobar las características genotípicas de la actividad física, ejemplo de esto, son los estudios realizados por Abramova, Nikitina y Ozolin (2013) quiénes encontraron diferentes marcas dermatoglificas en distintos grupos deportivos, demostrando un perfil dermatoglífico desigual por la capacidad física presentada. Esto demuestra que las características dermatoglíficas pueden considerarse, desde el punto de vista, biológico, indicativas del rendimiento en el terreno deportivo (Del Vecchio y Gongalves, 2011).

El handball es un deporte de equipo, se juega con una pelota, está conformado por 15 jugadores, solo siete juegan en el terreno, seis en el campo y un portero, el resto entran de cambio. Se juega en un terreno rectangular, con una portería a cada lado del campo. El objetivo del juego es desplazar la pelota a través del terreno de juego, valiéndose fundamentalmente de las manos, para intentar introducirla en la portería contraria, acción que se denomina gol (Tolano, Serna, Toledo, y Gavotto, 2015). En este deporte, se realizan muchos cambios de esfuerzos a elevada intensidad y corta duración con reducidos momentos de descanso. Durante estos esfuerzos los jugadores realizan muchas habilidades motoras específicas como correr, saltar, parar, fintar y lanzar, además de realizar acciones contra el adversario como empujes, golpeos, contactos o bloqueos (Ramos-Angulo et al., 2018).

Según Lagunes (2015), las capacidades físicas que predominan en este deporte son la resistencia aeróbica: para permitir mejores recuperaciones en un deporte de carácter intermitente, la fuerza explosiva de los miembros superiores e inferiores: para alcanzar altas velocidades de desplazamiento y lanzamiento, la fuerza máxima y la potencia: necesaria en las acciones de contacto contra los adversarios. Todas estas capacidades físicas son importantes para contribuir al alto rendimiento del equipo; al igual que para la realización de todas estas habilidades no sólo se depende de la fuerza muscular, sino también de otros factores como la coordinación de diferentes segmentos corporales (García et al., 2011; Michalsik, Madsen, y Aagaard, 2014). 
En México, el handball es un deporte relativamente nuevo, a pesar de ello ha tenido grandes avances en los últimos años (Lagunes, 2015). Sin embargo, hacen falta realizar estudios a profundidad para conocer las potencialidades físicas de los atletas de este deporte mediante la dermatoglifia las cuales ayudarán a los entrenadores y técnicos deportivos a mejorar su rendimiento y también realizar una buena orientación y detección de sus practicantes. Las cuales no se han realizado ningún trabajo al respecto.

Por ende, conocer el perfil dermatoglífico de los jugadores de handball de la ciudad de Chihuahua va a permitir saber si los deportistas cuentan con las características necesarias para hacer frente a las exigencias que demanda este deporte.

\section{Objetivo}

Identificar las potencialidades físicas más relevantes de los atletas universitarios masculinos de handball de la UACH a través del método dermatoglífico informatizado.

\section{Materiales y Métodos}

Este es un estudio exploratorio, transversal y descriptivo. En cuanto a la muestra, el entrenador y los deportistas fueron informados verbalmente sobre el objetivo y la naturaleza del estudio, la mayoría mostraron su aprobación para formar parte del mismo (consentimiento informado), la muestra fue escogida de forma intencionada, se invitaron a 20 jugadores masculinos del equipo de handball de la $U A C H$, donde solo asistieron a las mediciones un total de diez $(n=10)$ atletas con una edad promedio de 20.4 años, los cuales han participado en eventos nacionales y universitarios con resultados satisfactorios.

\section{Instrumento}

El protocolo escogido para conocer el potencial genético por medio de las impresiones digitales fue el dermatoglífico propuesto por Cummins e Midlo (1961). Para la captura, procesamiento y análisis de estas impresiones digitales fue utilizado el método informatizado, validado por Nodari-Júnior (2009) y Nodari y Heberle (2014) por medio del lector biométrico digital (Watson Mini), que está constituido por un escáner óptico de rodamiento que recolecta e interpreta las imágenes y construye en código binario un diseño que es capturado por un software específico que reconstruye las imágenes reales y binarias en blanco y negro. Después de tener recogidas todas las imágenes, el usuario del lector las selecciona una por una donde traza una línea llamada (línea de Galton) uniendo los puntos (núcleo y deltas) para que este software por medio de algoritmos específicos haga la intersección de la línea trazada entre los puntos con las líneas de la huella digital. Esto nos da a conocer la cantidad de líneas de cada uno de los dedos y el tipo de dibujo. El software hace una identificación cualitativa de la imagen y cuantitativa de la cantidad de líneas, formando la hoja informatizada que muestra los datos procesados (Alberti et al., 2018).

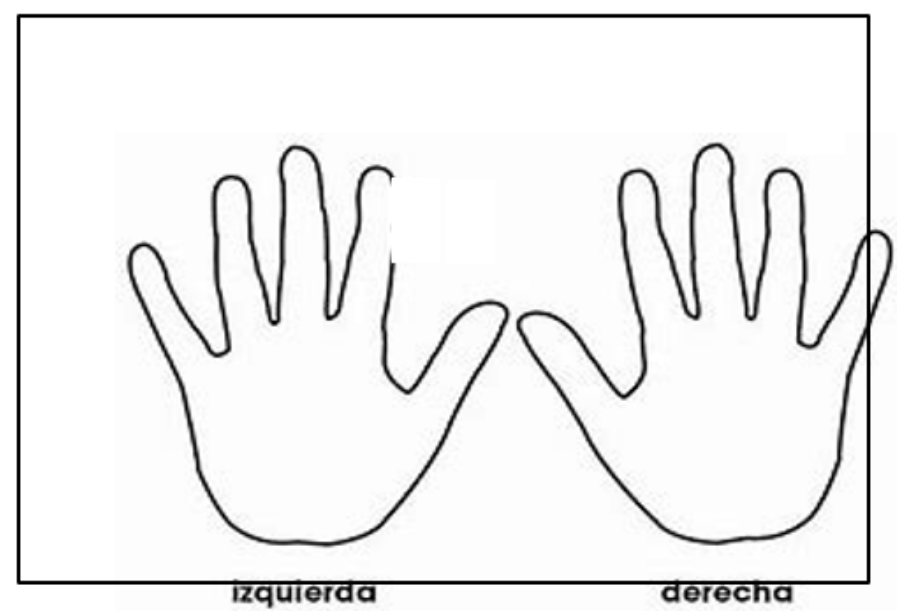

Figura 2. Orden numérico de los dedos, mano izquierda, dedo 1 pulgar (MET1), dedo 2 índice (MET2), dedo 3 medio (MET3), dedo 4 anular (MET4) y dedo 5 meñique (MET5) y de la mano derecha, dedo 1 pulgar (MDT1), dedo 2 índice (MDT2), dedo 3 medio (MDT3), dedo 4 anular (MDT4) y dedo 5 meñique (MDT5). Fuente: Nodari y Heberle (2014).$$
\text { C } 2 \text {. Nody }
$$ 
Análisis de datos

Los análisis estadísticos se procesaron utilizando el Paquete Estadístico para las Ciencias Sociales (SPSS), versión 18.0, los cuales consistieron en estadística descriptiva.

\section{Resultados}

De acuerdo con los resultados obtenidos sobre los aspectos cualitativos y cuantitativos a través de la frecuencia de los dibujos dermatoglíficos $A, L$ y W, se pueden ver los tipos de impresiones digitales presentes en cada uno de los dedos de las manos, prevaleciendo de forma general el dibujo presilla con una media de 7.9, luego el patrón verticilo con una media de 1.7 y mucho más reducida aún la presencia de arco con 0.4. Presentando el grupo de atletas estudiado predisposición innata a la velocidad y en menor medida de coordinación motora y agilidad, observada por la presencia de los patrones descritos anteriormente. En cuanto a los valores cuantitativos el D10 alcanzó un promedio de 11.3, representando para este grupo gran predisposición a la velocidad (Tabla 1).

Tabla 1.
En lo que corresponde a las características cuantitativas de la suma de líneas de la mano izquierda (SQTLE), la derecha (SQTLD) y el total de las dos manos (SQTL) se aprecia como media un mayor número de líneas en la mano derecha (56.9) que en la izquierda (53.5) y una media total de (110.4) con predisposición a la velocidad y la fuerza explosiva al igual que él (D10) debido a sus valores medios (Tabla 2).

Tabla 2.

Suma de líneas mano izquierda, derecha y total

\begin{tabular}{cccc}
\hline SUJETOS & SQTLE & SQTLD & SQTL \\
\hline 1 & 53 & 41 & 94 \\
2 & 58 & 63 & 121 \\
3 & 42 & 60 & 102 \\
4 & 65 & 71 & 136 \\
5 & 44 & 32 & 76 \\
6 & 32 & 45 & 77 \\
7 & 80 & 82 & 162 \\
8 & 51 & 51 & 102 \\
9 & 74 & 73 & 147 \\
10 & 36 & 51 & 87 \\
Media & 53.5 & 56.9 & 110.4 \\
Desv. Estándar & 15.1 & 14.9 & 28.3 \\
\hline
\end{tabular}

Nota: SQTLE: sumatoria de las líneas de la mano izquierda, SQTLD: sumatoria de líneas de la mano derecha, SQTL: sumatoria de líneas de las dos manos.

Datos cualitativos, cuantitativos y formulas dermatoglíficas

\begin{tabular}{|c|c|c|c|c|c|c|c|c|c|c|c|c|c|c|c|c|c|}
\hline SUJETO & MET1 & MET2 & MET3 & MET4 & MET5 & MDT1 & MDT2 & MDT3 & MDT4 & MDT5 & A & LR & LU & W & WS & D10 & TIPO FD \\
\hline 1 & LU & $L R$ & LU & LU & LU & A & $L R$ & LU & LU & LU & 1 & 2 & 7 & 0 & 0 & 9 & $L>A$ \\
\hline 2 & W & LU & LU & LU & LU & W & LU & LU & LU & LU & 0 & 0 & 8 & 2 & 0 & 12 & $L>W$ \\
\hline 3 & WS & LU & LU & LU & LU & W & LU & LU & LU & LU & 0 & 0 & 8 & 1 & 1 & 12 & $L>W$ \\
\hline 4 & WS & LU & W & W & W & LU & W & LU & W & W & 0 & 0 & 3 & 6 & 1 & 17 & $W>L$ \\
\hline 5 & LU & LU & LU & LU & LU & LU & LR & LU & LU & LU & 0 & 1 & 9 & 0 & 0 & 10 & $10 \mathrm{~L}$ \\
\hline 6 & W & A & A & LU & LU & LU & LU & A & LU & LU & 3 & 0 & 6 & 1 & 0 & 8 & LAW \\
\hline 7 & WS & LR & LU & LU & LU & W & WS & LU & LU & LU & 0 & 1 & 6 & 1 & 2 & 13 & $L>W$ \\
\hline 8 & LU & LU & LU & LU & LU & LU & LU & LU & LU & LU & 0 & 0 & 10 & 0 & 0 & 10 & $10 \mathrm{~L}$ \\
\hline 9 & LU & LU & LU & LU & LU & LU & LU & LU & LU & LU & 0 & 0 & 10 & 0 & 0 & 10 & $10 \mathrm{~L}$ \\
\hline 10 & LU & LU & LU & W & LU & LU & LU & LU & W & LU & 0 & 0 & 8 & 2 & 0 & 12 & $L>W$ \\
\hline & & & & & & & & & & Media & 0.4 & 0.4 & 7.5 & 1.3 & 0.4 & 11.3 & \\
\hline & & & & & & & & & sviac & stándar & 0.9 & 0.7 & 2.0 & 1.7 & 0.7 & 2.4 & \\
\hline
\end{tabular}

Nota: MET1: Dedo 1 mano izquierda, MET2: Dedo 2 mano izquierda, MET3: Dedo 3 mano izquierda, MET4: Dedo 4 mano izquierda, MET5: Dedo 5 mano izquierda, MDT1: Dedo 1 mano derecha, MDT2: Dedo 2 mano derecha, MDT3: Dedo 3 mano derecha, MDT4: Dedo 4 mano derecha, MDT5: Dedo 5 mano derecha, A: arco, LR: presilla radial, LU presilla ulnar, W: verticilo, WS: verticilo en forma de S, D10: sumatoria de líneas de los dedos de las dos manos. 
En cuanto al tipo de fórmulas dermatoglíficas, se encontró que la mayor prevalencia fue la de $L>W$ presente en 4 deportista $(40 \%)$, representando a la velocidad y buena coordinación motora, uno más de ellos cuenta con la formula $W>L$, es decir mayor coordinación motora, seguida de una buena velocidad, otro muestra la formula $L>A$, velocidad y fuerza (potencia) y finalmente el último tiene la fórmula dermatoglífica LAW, que se relaciona con un equilibrio en velocidad, fuerza y coordinación motora (Tabla 3).

Tabla 3.

Frecuencia y porcentajes de fórmulas dermatoglíficas
FORMULAS

DERMATOGĹFICAS

$10 \mathrm{~L}$

$\mathrm{L}>\mathrm{W}$

$W>L$

$L>A$

LAW
3

4

1

FRECUENCIA

1
$40 \%$

$110 \%$

$1 \quad 10 \%$
PORCENTAJES

$30 \%$

$10 \%$ por último una discreta presencia de fuerza pura (Nodari y Heberle, 2014). En relación al predominio de la velocidad, estos resultados se asemejan a los encontrados en el equipo femenino de balonmano brasileño, convocado para el Panamericano 2003 (Nogueira et al., 2005), pues presentan un buen nivel de velocidad como ocurre con los resultados del estudio de la selección femenina brasileña adulta de handball (da Cunha et al., 2006). Pero con mayor presencia de potencia que coordinación motora en los dos estudios con una predisposición genética para la capacidad de potencia, estos resultados, según los autores, se traducen en las características del balonmano femenino de alto rendimiento en Brasil.

Según Abramova (1995) corroborado por Castanhede (2003) entre otros autores, sus resultados no son típicos del alto rendimiento deportivo debido a la alta presencia del patrón (A), lo cual difieren de este estudio, también esto se puede relacionar con el sexo ya que los estudios del handball en Brasil se han realizado en atletas femeninas en comparación con este trabajo que es con hombres.

Nota: D10: total de deltas en los 10 dedos, $\mathrm{L}>\mathrm{W}$ : mayor presilla que verticilo, W>L: mayor verticilo que presilla, L>A: mayor presilla que arcos, LAW: presencia de presilla, arco y verticilo.

\section{Discusión}

Al analizar el tipo de diseño, a partir de la tabla de potencialidades físicas propuesta por los autores del software dermatoglífico, los atletas estudiados ostentan: de muy buena a excelente velocidad, seguidos de una satisfactoria o buena coordinación motora y
En otro estudio realizado con atletas de handball de playa también en Brasil (Alves et al., 2012) en relación a las variables cualitativas sus resultados corroboran los hallazgos de esta investigación fundamentalmente en los de las atletas de alto rendimiento donde presentan una velocidad de muy buena a excelente, una coordinación motora de regular a buena y una discreta presencia de fuerza pura. Hay que destacar en estos estudios la prevalencia de un número elevado de presillas, que indica la presencia de la capacidad física velocidad. Moreno (1996), en lo que respecta a esta capacidad en 
el handball, expresa que la misma es determinante en las acciones decisivas del juego tanto ofensivas como defensivas. Por lo antes expuesto es de vital importancia la velocidad en el handball por tratarse de un deporte colectivo donde su juego se basa en acciones intensas y dinámicas.

En cuanto a los valores de D10 y la sumatoria de la cantidad de líneas de las dos manos se observan bajos resultados en los estudios de Nogueira et al. (2005) y Castanhede (2003) en relación a esta investigación. En este aspecto, Dantas y Fernandes (2002) resaltan que valores bajos de D10 están relacionados a bajos niveles de predisposición a la coordinación. Cabe destacar que las acciones colectivas en los deportes con pelotas se caracterizan por un mayor grado de complejidad, por lo que se necesita un nivel más elevado de coordinación y de velocidad, cualidades físicas poco encontradas en las atletas Brasileñas.

En lo que consiste a las fórmulas dermatoglificas, al observar la combinación de dibujos corroboramos lo escrito por (Silva, 2004), donde expresan que cuando hay presencia (ALW) predisposición genética para la fuerza explosiva, $(\mathrm{L}>\mathrm{W})$ velocidad y $(\mathrm{W}>\mathrm{L})$ agilidad, son cualidades físicas inherentes a la práctica del balonmano, comprobando así que el grupo evaluado tiene características genotípicas relacionadas con la capacidad anaeróbico aláctico. Aunque el balonmano se identifica por la capacidad aeróbica, debido a su duración y por la intensidad del esfuerzo a lo largo del juego, la capacidad anaeróbica es la más significativa para esta modalidad deportiva, Dado que gran parte del movimiento predominante de los jugadores implica esfuerzos muy intensos y de corta duración. A pesar de que la velocidad es la capacidad física con mayor predisposición genética.

\section{Conclusiones}

Las potencialidades que más resaltan en este grupo de atletas de handball son la velocidad y la coordinación motora elementos inherentes para este y otros deportes colectivos. Hacen falta realizar estudios a profundidad para conocer las potencialidades físicas de los atletas de este deporte mediante la dermatoglifia, las cuales ayudarán a los entrenadores y técnicos deportivos a mejorar su rendimiento y también realizar una buena orientación y detección de sus practicantes. Por último, se reconoce que la muestra de este estudio es pequeña, se recomienda realizar más investigaciones con muestras mayores para corroborar los hallazgos obtenidos.

\section{Agradecimientos}

Agradecemos al equipo de handball y entrenador de la UACH Universidad Autónoma del Estado Chihuahua, por las facilidades otorgadas para poder llevar a cabo este estudio y a Salud: Dermatoglifia (DeSalud, A.C.) por la asesoría técnica recibida y por el apoyo sobre la utilización gratuita del lector dermatoglífico computarizado, institución que ostenta derecho de uso del mismo.

\section{Limitaciones}

Los resultados de este estudio no se pueden generalizar por la pequeña cantidad de la muestra ya que no todos los atletas quisieron participar de dicha investigación. Otra limitación fue la escasa evidencia de investigación científica en este deporte directamente relacionada con la dermatoglifia. 


\section{Referencias}

Abramova, T.; Nikitina, T.; Ozolin, N. (2013). De l'utilisation des dermatoglyphes digitaux dans la selection des sportifs. Teor Prak Fiz Kult. Vol. 3. p. 10-15.

Alberti, A., Fin, G., Gomes de Souza, R., Hur, B., y Nodari, R. J. (2018). Dermatoglífia: as impressões digitáis como marca característica dos atletas de futsal feminino de alto rendimento do Brasil. RBFF-Revista Brasileira de Futsal e Futebol, 10(37), 193-201. Recuperado de https://dialnet.unirioja.es/servlet/articulo? codigo $=6681228$

Alves, J. E., Marques, A. L., Mimbacas, A., y Gomes, U. M. (2012). Dermatoglifia, somatotipo e composição corporal no beach handball: Estudo comparativo entre diferentes níveis de qualificação esportiva. Motricidade, 8(2), 567-576.

Castanhede, A.; Fernandes, J. \& Dantas, P. (2003). Dermatoglyphic and Somatotype profile of male soccer athletes of high performance in Rio de Janeiro-Brazil. Fitness \& Performance Journal, 2(4), 234-239.

da Cunha, A. T., Pretto, A. C., Trevisan, A., \& Silva, P. M. (2006). Características dermatoglíficas, somatotípicas, psicológicas e fisiológicas da seleção brasileira feminina adulta de handebol. Fitness \& Performance Journal, 5(2), 81-86.

Del Vecchio, F. B.; Gonçalves, A. (2011). Dermatoglifos como indicadores biológicos del rendimiento deportivo. Rev Andal Med Deporte, 4(1), 44-52.
García, M., Alcaraz, P. E., Ferragut, C., Manchado, C., Abraldes, J. A., Rodríguez, N., y Vila, H. (2011). Composición corporal y velocidad de lanzamiento en jugadoras de élite de balonmano. Cultura, ciencia y deporte: revista de ciencias de la actividad física y del deporte de la Universidad Católica de San Antonio, (17), 129-136.

Hernández C. A., y Naranjo, R. A. (2016). Determinación del perfil genotípico y fenotípico en jugadoras bogotanas del club gol star. Revista digital: Actividad Física y Deporte, 2(1).

Hernández, M. (2017). Estudios de los dermatoglifos en fueguinos. Magallania (Punta Arenas), 45(1), 85-100.

Lagunes, J. O. (2015). Características cineantropométricas de la selección mexicana varonil de balonmano (Doctoral dissertation, Facultad de Organización Deportiva).

Michalsik LB, Madsen K, Aagaard P. (2014) Match performance and physiological capacity of female elite team handball players. Int J Sports Med.; 35(7):595-607.

Nodari, R.J. y Heberles, A. (2014). Leitor Dermatoglífico, Gold Standard de la dermatoglifia. (En línea). Recuperado de http://salusdermatoglifia.com.br/

Nodari-Júnior, R. J. (2009). Prototipo de escaneamento informatizado: possibilidade em diagnóstico em saúde por meio das impressões digitais. 2009. 58 (Doctoral dissertation, Tese (Doutorado-Ciências da Saúde) -Universidade Federal do Rio Grande do Norte, Natal). 
Nogueira, T. N., da Cunha, A. T., Silva, P. M., \& Fernandes, J. (2005). Perfil somatotípico, dermatoglífico e das qualidades físicas da seleção brasileira de handebol feminino adulto por posição de jogo. Fitness \& Performance Journal, 4(4), 236-242.

Ramos-Angulo, A. B., Medina-Porqueres, I., Ortiz-Bish, A., Ruiz-Martínez, Y., Medina-Jiménez, L., \& Elena-Gamboa, J. (2018). Perfil antropométrico de jugadoras de balonmano femenino de élite. Revista Andaluza de Medicina del Deporte, 11(2), 47-51.

Rodrigues, M., \& Fernandes Filho, J. (2004). Perfis dermatoglífico, somatotípico e das qualidades físicas básicas dos para-quedistas do exército brasileiro do ano de 2003. Fitness $\&$ Performance Journal, 3(2), 88-97.

Silva, P.M. (2004). Relação entre estado e predisposição genética no futsal brasileiro. Tese de Doutorado, Universidade Federal do Rio Grande do Norte, Natal, Brasil.

Tolano, E. J., Serna, A., Toledo, J., y Gavotto, O. I. (2015). Programa metodológico para la enseñanza de la técnica del balonmano en jugadores de 12 a 16 años. Lecturas: Educación física y deportes, (206), 12. 